PROCEEDINGS OF THE AMERICAN MATHEMATICAL SOCIETY

Volume 124, Number 10, October 1996

\title{
BOUNDARIES OF ROTATION SETS FOR HOMEOMORPHISMS OF THE $n$-TORUS
}

\author{
RICHARD SWANSON AND RUSSELL WALKER
}

(Communicated by Linda Keen)

\begin{abstract}
We construct a $C^{\omega}$ diffeomorphism of the 3 -torus whose rotation set is not closed. We prove that the rotation set of a homeomorphism of the $n$-torus contains the extreme points of its closed convex hull. Finally, we show that each pseudo-rotation set is closed for torus homeomorphisms.
\end{abstract}

\section{INTRODUCTION}

H. Poincaré introduced the idea of the rotation set of an orientation preserving circle homeomorphism and proved that it consists of a single real number $([\mathrm{P}])$. In 1979 S. Newhouse, J. Palis, and F. Takens introduced the idea of a rotation set for maps of the circle (homotopic to the identity) and proved that this set is always a closed interval ([N-P-T]). M. Handel has shown that the analogous rotation set for an annulus homeomorphism (isotopic to the identity) must be closed $([\mathrm{H}])$. It follows from work of J. Franks (but see also [M-Z]) that the rotation set of a 2-torus homeomorphism must contain the extreme points and the (2-dimensional) interior of its closed convex hull $([\mathrm{F}])$. (We extend this extreme point containment result to arbitrary $n$-tori in Theorem 4.8, using a functional analytic approach.)

Whether or not the rotation set of a 2-torus homeomorphism must be closed remains an open question. But M. Misiurewicz and K. Ziemian have shown that vectors on the boundary of the rotation set of a homeomorphism $f: \mathbb{T}^{2} \rightarrow \mathbb{T}^{2}$ need not be the expected value of the displacement by $f$ for any ergodic invariant probability measure $([\mathrm{M}-\mathrm{Z}])$. We caution the reader that the definition of rotation set used here corresponds to the "point rotation set" in [M-Z].

Our main result is the construction of a $C^{\omega}$ diffeomorphism $F: \mathbb{T}^{3} \rightarrow \mathbb{T}^{3}$ isotopic to the identity with a lift $\widetilde{F}: \mathbb{R}^{3} \rightarrow \mathbb{R}^{3}$ with rotation set $\rho(\widetilde{F})$ which is not closed (Theorem 3.2). (Note: throughout this article lifts of a map $G$ to the universal cover will be written $\widetilde{G}$.) The rotation set of $\widetilde{F}$ is found explicitly which is unusual for non-trivial analytic examples of rotation behavior.

Next we analyze $\rho(\widetilde{F})$ by proving two theorems about the general nature of boundaries of rotation sets for homeomorphisms of $\widetilde{G}: \mathbb{R}^{n} \rightarrow \mathbb{R}^{n}(n \geq 1)$ which are lifts of toral maps. First we use the Krein-Milman theorem to show that $\rho(\widetilde{G})$ contains the extreme points of its closed convex hull (Theorem 4.8). And second

Received by the editors April 4, 1995.

1991 Mathematics Subject Classification. Primary 58J22.

Key words and phrases. Rotation vectors on tori.

Research supported in part by NSF-OSR grant \#9350546. 
we show that the "pseudo-rotation set" (defined rigorously below) $\rho_{\psi}(\widetilde{G})$ (which contains $\rho(\widetilde{G})$ ) must be closed (Theorem 5.1). Roughly speaking, the latter set measures the rotation set that a computer might calculate. Such pseudo-rotation vectors are easy to compute and behave well on chain transitive sets, where they constitute a closed convex set. See [B-S] for more on pseudo rotation sets for annulus homeomorphisms. Lastly, we exhibit the pseudo rotation set, $\rho_{\psi}(\widetilde{F})$, for the example constructed above. Our work with rotation sets of 3 -torus homeomorphisms has led us to the following conjecture:

Conjecture 1.1. The rotation set of a lift of a 3-torus homeomorphism, isotopic to the identity, contains the closure of its (3-dimensional) interior.

\section{Definitions}

For general definitions consult an introductory text on dynamical systems such as [G-H]. We will take as our model of the $n$-torus the space $\mathbb{T}^{n}=\overbrace{S^{1} \times \cdots \times S^{1}}^{n}$, where $S^{1}=\{z \in \mathbb{C}:|z|=1\}$. As universal cover we will use the map $\Pi: \mathbb{R}^{n} \rightarrow \mathbb{T}^{n}$ given by $\Pi\left(x_{1}, \ldots, x_{n}\right)=\left(\exp 2 \pi i x_{1}, \ldots, \exp 2 \pi i x_{n}\right)$.

Now assume that $G: \mathbb{T}^{n} \rightarrow \mathbb{T}^{n}$ is isotopic to the identity and $\widetilde{G}: \mathbb{R}^{n} \rightarrow \mathbb{R}^{n}$ is a (continuous) lift of $G$. Then $\rho(\widetilde{G}, x)$ denotes the rotation set of $x \in \mathbb{R}^{n}$ under $\widetilde{G}$, and is given by

$$
\rho(\widetilde{G}, x)=\operatorname{LIM}\left\{\frac{\widetilde{G}^{n}(x)-x}{n} \mid n \in \mathbb{N}\right\} .
$$

Here we use "LIM" to denote "the set of limit points". The rotation set of $\widetilde{G}$ is the union

$$
\rho(\widetilde{G})=\bigcup_{x \in \mathbb{R}^{n}} \rho(\widetilde{G}, x) .
$$

For any map $f: X \rightarrow X$ on a compact metric space, let $\mathcal{M}_{I}(f)$ denote the set of $f$-invariant Borel probability measures, which forms a compact convex subset in the topological vector space of signed (regular Borel) measures on $X$. If $G: \mathbb{T}^{n} \rightarrow \mathbb{T}^{n}$, with a lift $\widetilde{G}$, define $\mathcal{M}_{I}(\widetilde{G})$ to be the set of lifts of regular Borel probability measures on $\mathbb{T}^{n}$. A Borel measure $\tilde{\mu}$ on $\mathbb{R}^{n}$ is the lift of the probability measure $\mu$ on $\mathbb{T}^{n}$ if for each fundamental domain $Q$ and Borel set $E \subset \mathbb{R}^{n}, \tilde{\mu}(E \cap Q)=$ $\mu(\Pi(E \cap Q))$. For example, the Haar measure on $\mathbb{T}^{2}$ lifts to Lebesgue measure on $\mathbb{R}^{2}$.

Define the mean rotation number by

$$
\rho_{\tilde{\mu}}(\widetilde{G})=\int_{Q}(\widetilde{G}-I d) d \tilde{\mu},
$$

where $Q$ is an arbitrary fundamental domain in the covering space of $\mathbb{T}^{n}$. When $\mu$ is ergodic for $G$, we will speak of the ergodic mean rotation number. Note that if $\mu$ is a $G$-invariant Borel probability measure on $\mathbb{T}^{n}$, then $\tilde{\mu}$ is a $\widetilde{G}$-invariant Borel (infinite!) measure on $\mathbb{R}^{n}$ that is invariant under deck translations.

Now define the mean rotation set

$$
\rho_{\operatorname{mes}}(\widetilde{G})=\bigcup_{\mu \in \mathcal{M}_{I}(\widetilde{G})} \rho_{\tilde{\mu}}(\widetilde{G})
$$


Define an $\epsilon$ pseudo-orbit to be an infinite sequence $\left\{z_{k}\right\}_{k \geq 0} \subset \mathbb{R}^{n}$ such that $\left\|z_{k+1}-\widetilde{G}\left(z_{k}\right)\right\|<\epsilon$ for $k \geq 0$. An $\epsilon$-chain is a finite sequence $z_{0}, z_{1}, \ldots, z_{m}$ with $\left\|z_{k+1}-\widetilde{G}\left(z_{k}\right)\right\|<\epsilon$ for all $k$ with $0 \leq k<m$. Define the pseudo-rotation set of the $\epsilon$ pseudo-orbit $\left\{z_{k}\right\}$ by $\rho_{\psi}\left(\left\{z_{k}\right\}\right)=\operatorname{LIM}\left\{\left(z_{k}-z_{0}\right) / k: k \in \mathbb{N}\right\}$. The union over all such pseudo-rotation sets, for a given $\epsilon$, is the $\epsilon$ pseudo-rotation set, $\rho_{\psi}(\widetilde{G}, \epsilon)$. Then $r \in \mathbb{R}^{n}$ is a pseudo-rotation vector if it lies in $\rho_{\psi}(\widetilde{G}, \epsilon)$ for each $\epsilon$. The analogous pseudo-rotation set for an annulus homeomorphism, in fact, equals the closure of the rotation set $([\mathrm{B}-\mathrm{S}])$. Thus, $\rho_{\psi}(\widetilde{G})=\rho(\widetilde{G})$, since M. Handel has shown that the true rotation set is closed $([\mathrm{H}])$. It is not hard to find examples of lifts, $\widetilde{G}$, of homeomorphisms of $\mathbb{T}^{2}$ for which $\rho(\widetilde{G}) \neq \rho_{\psi}(\widetilde{G})$.

We need these next definitions to state our theorem concerning extreme points. Let $X$ be a subset of a topological vector space $\mathbb{E}$. An extreme point $p \in X$ is any point which meets the following criterion: if $p=t x+(1-t) y$ for points $x$ and $y$ in $X$ and $0<t<1$, then $x=p=y$. A subset $C$ of a topological vector space is convex if whenever $x, y \in C$ then $t x+(1-t) y \in C$, for all $0 \leq t \leq 1$. A topological vector space $\mathbb{E}$ is locally convex if zero has a neighborhood base of convex sets. The closed convex hull $\langle X\rangle$ of $X$ is the intersection of all closed convex sets containing $X$. The closed convex hull equals the closure of the convex hull. The convex hull of $X$ equals the set of all finite linear combinations of the form $\sum t_{i} x_{i}$, where $x_{i} \in X$ and $\sum t_{i}=1$. If the linear space $\mathbb{E}$ is a metric space, then $\langle X\rangle$ consists of all convergent infinite series of the form $\sum t_{i} x_{i}$ with $x_{i} \in X$ for $i=1,2, \ldots$ and $\sum t_{i}=1$.

\section{A rotation SET Which IS NOT ClOSED}

We first construct a 1-parameter family of $C^{\omega}$ diffeomorphisms $\left\{f_{\lambda}: 0 \leq \lambda \leq\right.$ 1\} of $\mathbb{T}^{2}$ with lifts $\left\{\widetilde{f}_{\lambda}: \mathbb{R}^{2} \rightarrow \mathbb{R}^{2}\right\}$, for which the diameter of the rotation set is not continuous in the parameter. (No such family of circle maps or annulus homeomorphisms exists.) At $\lambda=\frac{1}{2},\left\{f_{\lambda} \mid \lambda \in[0,1 / 2]\right\}$ undergoes a double "blue sky" bifurcation. For $0 \leq \lambda<\frac{1}{2}, f_{\lambda}$ has no periodic orbits. And the rotation set of the lift $\rho\left(\tilde{f}_{\lambda}\right)=\left\{\left(\frac{1}{4}, \tau(\lambda)\right)\right\}$, where $\tau(\lambda)>0$ and $\tau(\lambda) \rightarrow 0$ as $\lambda \rightarrow \frac{1}{2}^{-}$. The map $f_{\frac{1}{2}}$ has a semi-stable orbit of period two and a parallel semi-stable fixed circle, and $\rho\left(\tilde{f}_{\frac{1}{2}}\right)=\left\{(0,0),\left(\frac{1}{2}, 0\right)\right\}$.

Each member of our family of lifts, $\left\{\tilde{f}_{\lambda}\right\}$, is the time-one map of a flow $\varphi_{\lambda}^{t}$ generated by the $C^{\omega}$ vector field $X_{\lambda}=\left(X_{\lambda}^{1}, X_{\lambda}^{2}\right)$ on $\mathbb{R}^{2}$, where

$$
X_{\lambda}^{1}(x, y)=\frac{1}{4}(1-\cos (2 \pi y)) \quad \text { and } \quad X_{\lambda}^{2}(x, y)=2-\cos (4 \pi y)-2 \lambda .
$$

Clearly the vectorfields $\left\{X_{\lambda}\right\}$ satisfy the following:

3.1) $X_{\lambda}(x, y)$ is independent of $x$.

3.2) $X_{\lambda}^{1}(x, y)=X_{\lambda}^{1}(y)$ is independent of $\lambda$, is an even function of $y$, and $X_{\lambda}^{1}(y)=$ $\frac{1}{2}-X_{\lambda}^{1}\left(\frac{1}{2}-y\right)$.

3.3) $X_{\lambda}^{2}(y)=X_{\lambda}^{2}\left(y+\frac{1}{2}\right)$.

Lemma 3.1. For all $0 \leq \lambda<\frac{1}{2}, \rho\left(\widetilde{f}_{\lambda}\right)=\left\{\left(\frac{1}{4}, \tau(\lambda)\right)\right\}$, where $\tau:\left[0, \frac{1}{2}\right] \rightarrow \mathbb{R}$ is a continuous non-increasing function such that $\tau(\lambda)=0$ if and only if $\lambda=\frac{1}{2}$. And when $\lambda=\frac{1}{2}, \rho\left(\widetilde{f}_{\lambda}\right)=\left\{(0,0),\left(\frac{1}{2}, 0\right)\right\}$. 
Proof. We will need the notation

$$
\rho_{x}\left(\widetilde{f}_{\lambda}\right)=\operatorname{proj}_{x} \rho\left(\widetilde{f}_{\lambda}\right) \text { and } \rho_{y}\left(\widetilde{f}_{\lambda}\right)=\operatorname{proj}_{y} \rho\left(\widetilde{f}_{\lambda}\right),
$$

where $\operatorname{proj}_{x}, \operatorname{proj}_{y}: \mathbb{R}^{2} \rightarrow \mathbb{R}$ are projections onto the $x$ - and $y$-axes. $\rho\left(\widetilde{f}_{\lambda}\right)(x, y)=$ $\rho\left(\varphi_{\lambda}^{t}\right)(x, y)$ for all $(x, y)([\mathrm{FM}])$. Thus by 3.1) $\rho\left(\widetilde{f}_{\lambda}\right)(x, y)$ is independent of $x$. Fix $\left(x_{0}, y_{0}\right)$ and let $\varphi_{\lambda}^{t}\left(x_{0}, y_{0}\right)=(x(t), y(t))$. So $\left(x^{\prime}(t), y^{\prime}(t)\right)=\left(X_{\lambda}^{1}, X_{\lambda}^{2}\right)$.

First assume $0 \leq \lambda<\frac{1}{2}$. Because $X_{\lambda}^{2}$ is a positive function only of $y$, by 3.1) $\rho\left(\tilde{f}_{\lambda}\right)(x, y)$ is independent of $(x, y)$. We first show $\rho_{x}\left(\widetilde{f}_{\lambda}\right)=\frac{1}{4}$ for $\lambda$ in this range. It follows from 3.3) that there exists $T>0$ (depending on $y_{0}$ ) such that

$$
y(t+T)=y+\frac{1}{2}
$$

for all $t$. Using 3.2) the reader should check that

$$
x(t)=x_{0}+\int_{0}^{t} X_{\lambda}^{1}(y(s)) d s=x_{0}+\frac{1}{2} t-\int_{T}^{t+T} X_{\lambda}^{1}(y(s)) d s .
$$

Thus

$$
x(t)=x_{0}+\frac{1}{2} t-x(t+T)+x(t)
$$

and

$$
\begin{aligned}
\rho_{x}\left(\widetilde{f_{\lambda}}\right) & =\operatorname{LIM}\left\{\frac{x(t)-x_{0}}{t} \mid t \geq 0\right\} \\
& =\operatorname{LIM}\left\{\frac{1}{2}-\frac{x(t+T)}{t}+\frac{x(T)}{t} \mid t \geq 0\right\} \\
& =\frac{1}{2}-\operatorname{LIM}\left\{\frac{x(t+T)-x_{0}}{t} \mid t \geq 0\right\} \\
& =\frac{1}{2}-\rho_{x}\left(\widetilde{f}_{\lambda}\right) .
\end{aligned}
$$

So $\rho_{x}\left(\widetilde{f}_{\lambda}\right)=\frac{1}{4}$ for $\lambda$ in this range.

Next we consider $\rho_{y}\left(\widetilde{f}_{\lambda}\right)$. The equation $y^{\prime}(t)=X_{\lambda}^{2}(y)$ generates a flow on $\mathbb{R}$. The time one map of this flow covers a circle homeomorphism with well-defined rotation number $([\mathrm{P}])$. In fact this rotation number is $\rho_{y}\left(\widetilde{f}_{\lambda}\right)$. In the statement of this lemma we call it $\tau(\lambda)$. But rotation numbers of circle homeomorphisms depend continuously on the homeomorphism. So $\tau$ is continuous. Now for each $y, X_{\lambda}^{2}(y)$ is a positive strictly decreasing function of $\lambda$ in this range. However, $X_{\frac{1}{2}}^{2}$ has a zero at $y=0$ (as well as many others). This means that $\rho_{y}\left(\tilde{f}_{\frac{1}{2}}\right)=0$ and consequently $\tau(\lambda) \rightarrow 0$ as $\lambda \rightarrow \frac{1}{2}$.

Now fix $\lambda=\frac{1}{2}$. Notice $X_{\frac{1}{2}}^{1}(x, y)>0$ and $X_{\frac{1}{2}}^{2}(x, y)$ has singularities only at $\left\{\frac{n}{2} \mid n \in \mathbb{Z}\right\}$. In terms of the torus coordinates $(\underline{x}, \underline{y})=\left(e^{2 \pi i x}, e^{2 \pi i y}\right)$ on $\mathbb{T}^{2}$, the $\omega$-limit set under $f_{\frac{1}{2}}$ of each point in $\mathbb{T}^{2}$ is either contained in $\{\underline{y}=1\}$ or $\{\underline{y}=-1\}$. But, for all $x \in \mathbb{R}$ and $n \in \mathbb{Z}, X_{\frac{1}{2}}^{1}(x, n)=0$ and $X_{\frac{1}{2}}^{1}\left(x, n+\frac{1}{2}\right)=\frac{1}{2}$. Thus, $\rho\left(\widetilde{f}_{\frac{1}{2}}\right)=$ $\left\{(0,0),\left(\frac{1}{2}, 0\right)\right\}$ as desired.

We now define our $C^{\omega}$ 3-torus diffeomorphism: Let $\widetilde{F}: \mathbb{R}^{3} \rightarrow \mathbb{R}^{3}$ be given by

$$
\widetilde{F}(x, y, z)=\left(\widetilde{F}_{\tilde{h}(z)}^{1}(x, y), \tilde{f}_{\tilde{h}(z)}^{2}(x, y), z\right),
$$


where $\widetilde{f}_{\lambda}=\left(\widetilde{f}_{\lambda}^{1}, \widetilde{f}_{\lambda}^{2}\right)$ was defined above and $\tilde{h}: \mathbb{R} \rightarrow \mathbb{R}$ is given by

$$
\tilde{h}(z)=\frac{1}{4}[1-\cos (2 \pi z)] \text {. }
$$

Notice $\widetilde{F}$ covers a $C^{\omega} \operatorname{map} F$ on $\mathbb{T}^{3}$.

Theorem 3.2. There exists a $C^{\omega}$ diffeomorphism of $\mathbb{T}^{3}$, isotopic to the identity, with lift $\widetilde{F}: \mathbb{R}^{3} \rightarrow \mathbb{R}^{3}$ such that $\rho(\widetilde{F})$ is not closed.

Proof. On each 2-torus, $\left\{\underline{z}=e^{2 \pi i z}=\right.$ const $\}, F$ is the time one map of the $X_{h(z)^{-}}$ flow. Thus $F$ is a $C^{\omega}$ diffeomorphism.

We consider two cases, $z=\frac{1}{2}$ and $z \neq \frac{1}{2}$. When $z=\frac{1}{2}, \tilde{h}(z)=\frac{1}{2}$ and by Lemma $3.1, \rho\left(\tilde{f}_{\tilde{h}(z)}\right)=\left\{(0,0),\left(\frac{1}{2}, 0\right)\right\}$. So since $F$ is the identity in the third coordinate,

$$
\rho(\widetilde{F})(x, y, z)=\left\{(0,0,0),\left(\frac{1}{2}, 0,0\right)\right\} .
$$

When $z \neq \frac{1}{2}, \tilde{h}(z) \in\left[0, \frac{1}{2}\right)$. So by Lemma 3.1, $\rho\left(\widetilde{F}_{\tilde{h}(z)}\right)=\left(\frac{1}{4}, \tau(\tilde{h}(z))\right)$. Thus,

$$
\rho(\widetilde{F})(x, y, z)=\left\{\left(\frac{1}{4}, \tau(\tilde{h}(z)), 0\right)\right\} .
$$

We now combine the two cases. Recall that $\tau$ is non-increasing, $\tau(\lambda)>0$, and $\tau(\lambda) \rightarrow 0$ as $\lambda \rightarrow \frac{1}{2}$. Thus

$$
\rho(\widetilde{F})=\{(0,0,0)\} \bigcup\left\{\left(\frac{1}{2}, 0,0\right)\right\} \bigcup\left\{\left(\frac{1}{4}, y, 0\right) \mid 0<y<\tau(0)\right\},
$$

which is not closed.

Comments: By compactness, $\tau(0)>0$. A computer computation shows $\tau(0) \approx 1$. However, by rescaling the vector fields $\left\{X_{\lambda}\right\}$, we can arrange for $\tau(0)$ to equal any desired positive real number.

\section{EXtreme POINTS OF ROTATiON SETS}

In this section we prove that rotation sets of lifts of $n$-torus homeomorphisms $(n \geq 1)$ contain their extreme points. We need several theorems from functional analysis.

Theorem 4.1 (Krein-Milman). Suppose $\mathbb{E}$ is a locally convex topological vector space. A compact convex subset $X$ is the closed convex hull of the set of its extreme points.

Let $\mathcal{M}_{I}(\widetilde{G})$ denote the set of lifts of regular Borel $G$-invariant probability measures on $\mathbb{T}^{n}$ (as defined in Section 2).

Note that $\mathcal{M}_{I}(\widetilde{G})$ is simply the space of $\widetilde{G}$-invariant regular Borel measures on $\mathbb{R}^{n}$ that are invariant under deck translations and have the property $\tilde{\mu}(Q)=1$ for each fundamental domain $Q$.

There is considerable awkwardness in attempting to transport dynamical and measure theory arguments back and forth between a manifold and its covering space. One solution is to make all computations in the covering space. Towards this end, we shall identify the topological vector space $\mathcal{S}$ of signed measures on $\mathbb{T}^{n}$ with the space $\tilde{\mathcal{S}}$ of their lifted counterparts on $\mathbb{R}^{n}$. Topologize the measures via the customary separable and metrizable "weak* topology": a sequence $\left\{\tilde{\mu}_{n}\right\}$ in $\tilde{\mathcal{S}}$ converges to a measure $\tilde{\mu}$ if and only if the sequence of integrals $\int_{Q} \tilde{k} d \tilde{\mu}_{i}$ converges 
to $\int_{Q} \tilde{k} d \tilde{\mu}$ for each lift $\tilde{k}: \mathbb{R}^{n} \rightarrow \mathbb{R}$ of a map $k: \mathbb{T}^{n} \rightarrow \mathbb{R}$. By Alaoglu's Theorem, in this topology the space of probability measures is compact (e.g. [D-S], page 424).

We may summarize this discussion as the following lemma:

Lemma 4.2. Given a torus homeomorphism $G: \mathbb{T}^{n} \rightarrow \mathbb{T}^{n}$, the correspondence $\mu \mapsto \tilde{\mu}$ defines a canonical topological vector space isomorphism from the topological vector space $\mathcal{S}$ of signed (regular Borel) measures on $\mathbb{T}^{n}$ onto the space $\widetilde{\mathcal{S}}$ (of lifts of such signed measures) which carries $\mathcal{M}_{I}(G)$ onto $\mathcal{M}_{I}(\widetilde{G})$.

A proof of the following lemma may be found in [D-S, p. 428], for example.

Lemma 4.3. The space of signed measures $\mathcal{S}$, with the weak* topology is locally convex. Therefore, the space of lifts $\widetilde{\mathcal{S}}$ is locally convex.

Theorem 4.4 (Choquet's Theorem). A G-invariant Borel probability measure is an extreme point of $\mathcal{M}_{I}(G)$ if and only if it is ergodic. Hence, the extreme points of $\mathcal{M}_{I}(\widetilde{G})$ are lifts of ergodic measures on $\mathbb{T}^{n}$.

For a simple proof of the proceeding theorem in any compact metric space, consult [M, p. 104]. Define the lifted measure $\tilde{\mu}$ to be ergodic with respect to $\widetilde{G}$ if and only if $\mu$ is ergodic with respect to $G$.

Our next lemma is then a consequence of Theorems 4.1 and 4.4 and Lemmas 4.2 and 4.3 .

Lemma 4.5. The set of $\widetilde{G}$-invariant measures, $\mathcal{M}_{I}(\widetilde{G})$, is the closed convex hull of the (extreme point) set of ergodic $\widetilde{G}$-invariant measures.

Now suppose again that $\widetilde{G}: \mathbb{R}^{n} \rightarrow \mathbb{R}^{n}$ is a lift of an $n$-torus homeomorphism, $G$, and let $Q$ be a fundamental domain of $\mathbb{R}^{n}$. The following (as in [B-S]) is an easy application of the powerful Birkhoff Ergodic Theorem (for a proof, see [M, p. 89]):

Lemma 4.6. If $\tilde{\mu} \in \mathcal{M}_{I}(\widetilde{G})$ is ergodic, then

$$
\rho(\widetilde{G}, x)=\rho_{\tilde{\mu}}(\widetilde{G})=\int_{Q}(\widetilde{G}-I d) d \tilde{\mu}
$$

for $\tilde{\mu}$-almost all $x \in \mathbb{R}^{n} \cap Q$.

Proof. Apply the Birkhoff Ergodic Theorem to each component function of the vector field $\widetilde{G}-I d$ using the measure $\tilde{\mu}$ and the measure-preserving transformation $\widetilde{G}$.

Recall that $\rho_{\text {mes }}(\widetilde{G})$ is the union of all mean rotation vectors $\rho_{\tilde{\mu}}(\widetilde{G})$.

Lemma 4.7. The mean rotation set $\rho_{\mathrm{mes}}(\widetilde{G})$ is closed, convex and contains the rotation set, $\rho(\widetilde{G})$.

Proof. That $\rho_{\text {mes }}(\widetilde{G})$ is closed and convex follows from the fact that $\mathcal{M}_{I}(\widetilde{G})$ is closed and convex in the space of lifted signed measures on $\mathbb{R}^{n}$ and the map $\mu \mapsto \rho_{\mu}$ is linear and continuous. Now let $r \in \rho(\widetilde{G})(x)$. Then

$$
r=\lim _{i \rightarrow \infty}\left(\widetilde{G}^{n_{i}}(x)-x\right) / n_{i}
$$

for some subsequence of natural numbers, $n_{i} \rightarrow \infty$. Let $\tilde{\delta}(x)$ denote the Borel measure that assigns unit mass to each lattice point $x+\vec{n}$ for all $\vec{n} \in \mathbb{Z}^{n}$. Let $\tilde{\mu}$ be 
a limit point of the sequence of measures

$$
\tilde{\mu}_{i}=\sum_{j=1}^{n_{i}} \frac{1}{n_{i}} \tilde{\delta}\left(\widetilde{G}^{j}(x)\right) .
$$

Recall that the set $\mathcal{M}_{I}(\widetilde{G})$ of invariant measures is compact (hence, sequentially compact in the metrizable weak* topology). Then $\tilde{\mu}$ is $\widetilde{G}$-invariant and $\lim _{i \rightarrow \infty} \rho_{\tilde{\mu}_{i}}(\widetilde{G})=\rho_{\tilde{\mu}}(\widetilde{G})=r$.

Theorem 4.8. The rotation set $\rho(\widetilde{G})$ contains the extreme points of the mean rotation set, $\rho_{\text {mes }}(\widetilde{G})$. In particular, $\rho_{\text {mes }}(\widetilde{G})$ equals the closed convex hull of $\rho(\widetilde{G})$. Furthermore, $\rho(\widetilde{G})$ contains the extreme points of its closure. Finally, these extreme points correspond to ergodic mean rotation numbers.

Proof. Let $r$ be an extreme point of $\rho_{\operatorname{mes}}(\widetilde{G})$. Then $r=\rho_{\tilde{\mu}}(\widetilde{G})$ for some $\widetilde{G}$-invariant probability measure $\mu$. By Lemma $4.5, \mu$ lies in the closed convex hull of the $\widetilde{G}$ invariant ergodic measures. In particular, we can write $\mu=\sum t_{i} \mu_{i}$, where $\sum t_{i}=1$, $0 \leq t_{i} \leq 1$, and each $\mu_{i}$ is ergodic. (This sum may be infinite.) And $r=\rho_{\tilde{\mu}}(\widetilde{G})=$ $\sum t_{i} \rho_{\mu_{i}}(\widetilde{G})$.

If $\mu$ is not ergodic, we may assume, by reordering the index if necessary, that $0<$ $t_{1}<1$. In such case define the probability measures $\nu_{1}=\mu_{1}$ and $\nu_{2}=\frac{1}{M} \sum_{i \geq 2} t_{i} \mu_{i}$, where $M=\sum_{i \geq 2} t_{i}$. Then $r=\rho_{\tilde{\mu}}(\widetilde{G})=t_{1} \rho_{\widetilde{\nu_{1}}}+\left(1-t_{1}\right) \rho_{\widetilde{\nu_{2}}}$. But, by definition of extreme point, this only happens if $\rho_{\widetilde{\nu_{1}}}=\rho_{\tilde{\mu}}=\rho_{\widetilde{\nu_{2}}}$. So $\rho_{\tilde{\mu}}=\rho_{\widetilde{\mu_{1}}}$, where $\widetilde{\mu_{1}}$ is ergodic and by Lemma $4.6 \rho_{\widetilde{\mu_{1}}}(\widetilde{G}) \in \rho(\widetilde{G})$. The last two statements in the theorem follow from Lemma 4.7 .

\section{Pseudo-Rotation Sets ARe Closed}

The pseudo-rotation set $\rho_{\psi}(\widetilde{G}) \supset \rho(\widetilde{G})([\mathrm{B}-\mathrm{S}])$. In this section we prove that $\rho_{\psi}(\widetilde{G})$ is closed.

Theorem 5.1. Let $\widetilde{G}: \mathbb{R}^{n} \rightarrow \mathbb{R}^{n}(n \geq 1)$ be the lift of an $n$-torus homeomorphism. Then the pseudo-rotation set $\rho_{\psi}(\widetilde{G})$ is closed.

Proof. Assume that

$$
r \in \overline{\rho_{\psi}(\widetilde{G})}=\overline{\bigcap_{\epsilon>0} \rho_{\psi}(\widetilde{G}, \epsilon)} .
$$

Then $r$ is in $\overline{\rho_{\psi}(\widetilde{G}, \epsilon)}$ for all $\epsilon>0$. So for each $\epsilon>0$, there exist $\epsilon$ pseudo-orbits $\mathcal{O}_{k}$ such that $r=\lim _{k \rightarrow \infty} r_{k}(\epsilon)$, for some $r_{k}(\epsilon) \in \rho_{\psi}\left(\mathcal{O}_{k}\right)$.

We will construct a $2 \epsilon$ pseudo-orbit $\left\{z_{m}\right\}$ for $\widetilde{G}$ such that $\rho_{\psi}\left(\left\{z_{m}\right\}\right)=r$. Let $Q=[0,1]^{n}$ as before. By passing to a subsequence we may assume $\left\{\overline{\mathcal{O}_{n_{k}}} \cap Q\right\}$ converges (in the Hausdorff topology on compact subsets). By periodicity of the lift, the convergence holds for each choice $Q$ of fundamental domain, and, therefore, we can choose $k$ sufficiently large that each vector in $\mathcal{O}_{n_{k}}$ lies within $\epsilon$ of some vector in $\mathcal{O}_{n_{k+1}}$.

Now choose $\left\{z_{0}, z_{1}, \ldots, z_{m_{1}}\right\} \subset \mathcal{O}_{n_{1}}$ with $m_{1}$ large enough that

$$
\left\|\frac{z_{m_{1}}-z_{0}}{m_{1}}-r_{n_{1}}\right\|<\frac{1}{2} .
$$


Choose $z_{m_{1}+1} \in \mathcal{O}_{n_{2}}$ such that $\left\|z_{m_{1}}-z_{m_{1}+1}\right\|<\epsilon$. Next choose $m_{2} \gg m_{1}$ and an $\epsilon$-chain $\left\{z_{m_{1}+1}, \ldots, z_{m_{2}}\right\} \subset \mathcal{O}_{n_{2}}$ such that

$$
\left\|\frac{z_{m_{2}}-z_{0}}{m_{2}}-r_{n_{2}}\right\|<\frac{1}{4}
$$

Notice $\left\{z_{0}, z_{1}, \ldots, z_{m_{2}}\right\}$ is a $2 \epsilon$-chain for $\widetilde{G}$.

Continuing recursively, define $\left\{z_{m_{k}}\right\}$ and a $2 \epsilon$ pseudo-orbit $\left\{z_{0}, z_{1}, \ldots\right\}$ such that for all $k$,

$$
\left\|\frac{z_{m_{k}}-z_{0}}{m_{k}}-r_{n_{k}}\right\|<\frac{1}{2 k}
$$

Thus $\rho_{\psi}\left(\left\{z_{m}\right\}\right)=r$ and $r(\epsilon) \in \rho_{\psi}(\widetilde{G}, 2 \epsilon)$.

So, $r \in \rho_{\psi}(\widetilde{G})$ as desired.

To complete our investigation of the lift $\widetilde{F}$ of a 3-torus diffeomorphism constucted in Section 3, we now locate its pseudo-rotation set. An easy proposition is that the pseudo-rotation set of the restriction of $\widetilde{G}$ to the lift $\widetilde{\Lambda}$ of a chain transitive set $\Lambda$ in $\mathbb{T}^{n}$ is closed and convex. The reason is that one can construct pseudo-orbit itineraries for each $\epsilon>0$ that spend an arbitrary number of iterates on any desired true orbit. On the other hand an $\epsilon$-chain only requires a bounded number of iterates to join any two points of $\Lambda$. Thus, we have

Proposition 5.2.

$$
\rho_{\psi}(\widetilde{F})=\langle\rho(\widetilde{F})\rangle .
$$

Notes: 1) Notice $\rho(\widetilde{F})$ contains its extreme points (as required in Theorem 4.8). But $\rho(\widetilde{F})$ does not have any (non-empty) 3-dimensional interior nor the (non-empty) 2-dimensional interior of its convex hull.

2) Consider the Cartesian product of $\widetilde{F}$ with the identity map on $\mathbb{R}^{n-3}(n \geq 4)$. This map covers a $C^{\omega}$ diffeomorphism of $\mathbb{T}^{n}$. And the geometry of its rotation set is identical to that of $\widetilde{F}$.

3) Any compact subset of $\mathbb{R}^{n}$ can be realized as the rotation set of some diffeomorhism $F: \mathbb{T}^{2 n} \rightarrow \mathbb{T}^{2 n}$, using methods similar to the construction in this paper.

\section{REFERENCES}

[B-S] M. Barge and R. Swanson, Rotation shadowing properties of circle and annulus maps, Ergod. Thy. \& Dynam. Sys. 8 (1988), 509-521. MR 90f:58107

[D-S] N. Dunford and N. Schwartz, Linear Operators, Part I, Pure and Applied Math. Vol. VII, John Wiley and Sons, New York, 1957. MR 22:8302

[F] J. Franks, Rotation vectors for torus homeomorphisms, Trans. Am. Math. Soc. vol. 311, No. 1, (1989), 107-115. MR 89k:58239

[G-H] J. Guckenheimer and P. Holmes, Nonlinear Oscillations, Dynamical Systems, and Bifurcations of Vector Fields, Springer-Verlag, Applied Mathematical Sciences 42, New York, 1983. MR 85f:58002

[H] M. Handel, The rotation set of a homeomorphism of the annulus is closed, Commun. Math. and Phys. 127 (1990), 339-349. MR 91a:58102

[M] R. Mañé, Ergodic Theory and Differentiable Dynamics Springer-Verlag, New York, 1987. MR 88c: 58040

[M-Z] M. Misiurewicz and K. Ziemian, Rotation sets for maps of tori, J. London. Math. Soc. (2), vol. 40 (1989), 490-506. MR 91f:58052 
[N-P-T] S. Newhouse, J. Palis, and F. Takens, Bifurcations and stability of families of diffeomorphisms, Publ. Math. IHES, vol. 57 (1983), 5-71. MR 84g:58080

[P] H. Poincarè, "Étude particulière du tore", Chap. XV in Sur les courbes définies par les equations différentielles. III, J. Math. Pures Appl. (4) 1 (1885), 220-244; reprinted in Oeuvres de Henri Poincaré. Tome I, Gauthier-Villars, Paris, 1928, pp. 137-158.

Department of Mathematical Sciences, Montana State University, Bozeman, MonTANA 59717-0240

E-mail address: dswanson@math.montana.edu

E-mail address: walker@math.montana.edu 